\title{
Application research of three-dimensional transesophageal echocardiography in predicting prosthetic valve size for transcatheter aortic valve implantation
}

\author{
Xin Meng ${ }^{1 \#} \wedge$, Yandan Sun ${ }^{2 \#}$, Wei Bai ${ }^{1}$, Yuxi Li ${ }^{1}$, Shengjun Tuo ${ }^{1}$, Liang Cao ${ }^{1}$, Mengmeng Du ${ }^{1}$, Yang Liu ${ }^{3}$, \\ Ping Jin ${ }^{3}$, Jian Yang ${ }^{3}$, Liwen Liu ${ }^{1}$ \\ ${ }^{1}$ Department of Ultrasound, Xijing Hospital, Air Force Medical University, Xi'an, China; ${ }^{2}$ Department of Ultrasound, The 986th Hospital of the Air \\ Force, Xi'an, China; ${ }^{3}$ Department of Cardiovascular Surgery, Xijing Hospital, Air Force Medical University, Xi'an, China \\ Contributions: (I) Conception and design: L Liu, J Yang; (II) Administrative support: L Liu, J Yang, S Tuo, M Du; (III) Provision of study materials or \\ patients: W Bai, Y Li, L Cao, Y Liu, P Jin; (IV) Collection and assembly of data: X Meng, Y Sun, W Bai, Y Li; (V) Data analysis and interpretation: \\ X Meng, Y Sun, S Tuo; (VI) Manuscript writing: All authors; (VII) Final approval of manuscript: All authors. \\ \#These authors contributed equally to this work. \\ Correspondence to: Liwen Liu, PhD, MD. Department of Ultrasound, Xijing Hospital, Air Force Medical University, 127 Changle West Rd., Xi'an \\ 710032, China. Email: liwenfmmu@163.com; Jian Yang, PhD, MD. Department of Cardiovascular Surgery, Xijing Hospital, Air Force Medical \\ University, 127 Changle West Rd., Xi'an 710032, China. Email: yangjian1212@hotmail.com.
}

Background: Transcatheter aortic valve implantation (TAVI) is an alternative method to treat patients with severe aortic valve disease. Accurate measurement of the aortic valve annulus and selection of the appropriate artificial valve are critical to the success of TAVI. Multilayer spiral computed tomography (MSCT) is recommended as the "gold standard" for assessing the aortic valve annulus before TAVI. However, MSCT scanning may not be possible for patients with iodine allergy, renal failure, or pregnancy. The purpose of this study is to evaluate the aortic valve annulus by three-dimensional transesophageal echocardiography (3D-TEE) and compare the results with MSCT, exploring the feasibility of 3D-TEE to guide the selection of artificial valve implantation in TAVI.

Methods: We retrospectively analyzed 74 patients who successfully underwent TAVI in our hospital. Before the operation, 3D-TEE and MSCT were used to measure the maximum diameter, minimum diameter, areaderived diameter, and perimeter-derived diameter of the aortic valve annulus, and the results were analyzed for consistency. To predict the valve size based on 3D-TEE and the MSCT area-derived diameter, we compared the differences between the predicted valve size and the actual implanted valve size, and analyzed the differences between 3D-TEE and MSCT for guiding the selection of the prosthetic valve size.

Results: There was no significant difference between 3D-TEE and MSCT in the measurement of the maximum diameter, minimum diameter, area, and perimeter of the aortic annulus and their derived diameter $(\mathrm{P}>0.05)$. The intraclass correlation coefficients for the maximum diameter, minimum diameter, area-derived diameter, and perimeter-derived diameter of the aortic annulus were $0.89,0.83,0.84$, and 0.92 , respectively. There was no statistical difference in the accuracy of both methods, 3D-TEE and MSCT, in predicting different prosthetic valve sizes for TAVI $(\mathrm{P}>0.05)$.

Conclusions: 3D-TEE and MSCT have good agreement for measuring the values of various parameters of the aortic annulus. The accuracy of both methods was similar for predicting the aortic prosthetic valve size. 3D-TEE may provide guidance for selecting the prosthetic valve size for TAVI.

Keywords: Three-dimensional transesophageal echocardiography (3D-TEE); multilayer spiral computed tomography (MSCT); transcatheter aortic valve implantation (TAVI); valve annulus; prosthetic valve size

^ ORCID: 0000-0002-0063-7496. 
Submitted Nov 24, 2021. Accepted for publication Jan 18, 2022.

doi: $10.21037 / \mathrm{atm}-21-6577$

View this article at: https://dx.doi.org/10.21037/atm-21-6577

\section{Introduction}

For patients with severe aortic valve disease who cannot tolerate thoracotomy, transcatheter aortic valve implantation (TAVI) as an alternative method has achieved positive results (1). Accurate measurement of the aortic valve annulus and selection of the appropriate artificial valve are critical to the success of the TAVI procedure (2). Multilayer spiral computed tomography (MSCT) is recommended as the "gold standard" for assessing the aortic valve annulus before a TAVI operation because it can clearly show the threedimensional anatomical structure of the heart and blood vessels. However, MSCT scans may not be possible for patients with iodine allergies, renal failure, hyperthyroidism, or pregnancy. To solve this problem, finding a reliable alternative examination method to evaluate the aortic annulus and choose the prosthetic valve size is necessary. Previous study has shown that the evaluation of the aortic valve annulus by three-dimensional transesophageal echocardiography (3D-TEE) is in good agreement with MSCT (3). However, its guidance for prosthetic valve size selection is not yet supported by studies. This study retrospectively analyzed the $3 \mathrm{D}$-TEE measurement results of patients undergoing TAVI in Xijing Hospital and compared the results with the CT measurement and the actual selected prosthetic valve size. These results prove the feasibility of $3 \mathrm{D}$-TEE in guiding the implantation of artificial valves in TAVI operations. We present the following article in accordance with the STROBE reporting checklist (available at https://atm.amegroups.com/article/ view/10.21037/atm-21-6577/rc).

\section{Methods}

\section{Participants}

We retrospectively analyzed 74 patients who successfully underwent TAVI in Xijing Hospital from January 2019 to July 2020, including 48 men (64.9\%) and 26 women $(35.1 \%)$, with an average age of $68.11 \pm 7.38$ years. The inclusion criteria for TAVI surgery were as follows: (I) those with severe aortic stenosis or aortic regurgitation; (II) those with high risk factors and contraindications to surgery; (III) patients with significant chest pain, dyspnea, or other symptoms, as well as New York Heart Association (NYHA) cardiac function class II-IV, and (IV) life expectancy $>1$ year after aortic stenosis (AS) release. The exclusion criteria for TAVI surgery were as follows: (I) recent acute myocardial infarction; (II) mural thrombosis of the left ventricle; (III) severe left ventricular outflow tract obstruction; (IV) ascending aortic aneurysm at risk of rupture; (V) anatomy not suitable for TAVI surgery; and (VI) life expectancy $<1$ year. All patients were followed up for 1 year. All procedures in this study involving human participants were performed in accordance with the Declaration of Helsinki (as revised in 2013). This study was approved by the ethics board of Xijing Hospital (approval No. QX20191043-1). Informed consent was taken from all the patients.

\section{Apparatus and method}

Before the TAVI operation, all patients underwent MSCT (Siemens Somatom Definition Flash, Germany) for aortic scanning. The images were acquired using a retrospective cardiac gating method. The scan area was craniocaudal from the subclavian artery to the iliofemoral branches, with a scan layer thickness of $0.75 \mathrm{~mm}$ and a reconstruction layer thickness of $0.5 \mathrm{~mm}$. During the scan, $70 \mathrm{~mL}$ of iopromide $(370 \mathrm{mg} / \mathrm{mL})$ and $20-50 \mathrm{~mL}$ of physiological saline were injected via the elbow vein at a rate of $5.0 \mathrm{~mL} / \mathrm{s}$. The aortic annulus was measured at 30-40\% systolic phase, the lowest point of each sinus was marked in a double oblique position, and the aortic annular plane was automatically generated by the measurement software. The appropriate prosthetic valve size was preselected based on the mean diameter of the aortic annulus, and the selection criteria are shown in Tables 1,2. The Venus Medtech A-valve [Venus Medtech (Hangzhou) Inc., China], implanted via a femoral cannula, is recommended for AS, and the J-valve (Suzhou Jiecheng Medical Technology Co., Ltd., China), implanted via apical puncture, is recommended for aortic insufficiency (AI). Patients with both AS and AI need to be evaluated for the severity of both lesions. The A-valve is preferred for those with predominant AS, and the J-valve is preferred for those with predominant AI. It is also necessary to evaluate the structures above and below the annulus, such as the left ventricular outflow tract, ascending aorta, and the height 
Table 1 Venus Medtech A-valve selection criteria

\begin{tabular}{lcccc}
\hline Valve size & A23 $(18 \mathrm{Fr})$ & A26 (19 Fr) & A29 (19 Fr) & A32 (19 Fr) \\
\hline CT-diameter $(\mathrm{mm})$ & $17.0-19.9$ & $20.0-22.9$ & $23.0-25.9$ & $26.0-28.9$ \\
CT-perimeter $(\mathrm{mm})$ & $53.0-62.9$ & $63.0-71.9$ & $72.0-81.9$ & $82.0-90.9$ \\
CT-area $\left(\mathrm{mm}^{2}\right)$ & $227.0-313.9$ & $314.0-414.9$ & $415.0-530.9$ & $531.0-660.9$ \\
\hline
\end{tabular}

Table 2 J-valve selection criteria

\begin{tabular}{lccccc}
\hline Valve size & 21 & 23 & 25 & 27 & 29 \\
\hline CT-diameter $(\mathrm{mm})$ & $19.0-19.9$ & $20.0-21.9$ & $22.0-23.9$ & $24.0-25.9$ & $25.0-28.0$ \\
\hline
\end{tabular}
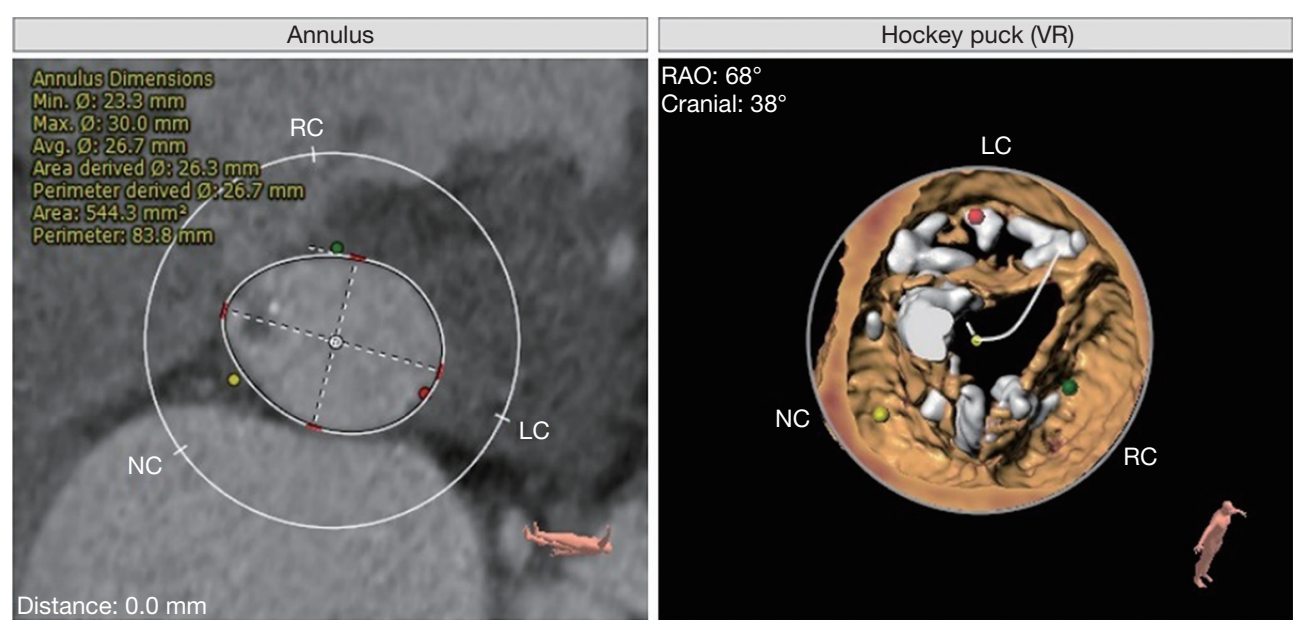

Figure 1 MSCT measurement of the aortic valve annulus parameter. MSCT, multilayer spiral computed tomography.

of the coronary artery opening, to make a comprehensive choice based on the key factors of minimally invasive, safe, and effective.

The patient was placed in the supine position using a Philips IE Elite Doppler ultrasound system, including an $\mathrm{X} 7-2$ 3D transesophageal probe with a probe frequency of 2-7 MHz, Qlab3DQ analysis software, and a connecting electrocardiogram (ECG) lead. After the patient was under general anesthesia, the TEE probe was sent into the middle part of the esophagus at a depth of approximately $35 \mathrm{~cm}$. Short-axis images of the aortic root were acquired at approximately $30-60^{\circ}$ in the middle of the esophagus, and $3 \mathrm{D}-\mathrm{ZOOM}$ imaging was used to reconstruct the $3 \mathrm{D}$ ultrasound image of the aortic root. We adjusted the 3 mutually perpendicular planes so that the cross-sectional plane just passed through the plane of the lowest point of the aortic valve leaflet attachment, and the resulting section was the aortic annulus plane. Qlab3DQ analysis software was applied to measure the maximum and minimum diameters of the aortic annulus as well as the area and circumference of the aortic annulus, and the diameter of the annulus was derived from the circular area formula and the perimeter formula. The correlation between the 2 derived diameters of TEE and CT was compared, and the valve size was selected according to the area-derived diameter $(4,5)$. Intraoperative $3 \mathrm{D}-\mathrm{TEE}$ was used throughout to assist the puncture sheath across the aortic valve. The position and angle of valve release were observed in real time, and the occurrence of perivalvular leaks and other complications in the immediate postoperative period were monitored. 3D-TEE was performed and analyzed by 2 experienced ultrasonographers, and the average was taken after 3 measurements per case.

A double-blind method was implemented to obtain 3D-TEE and MSCT measurements separately. Intraoperatively, valve dimensions were predicted by MSCT 

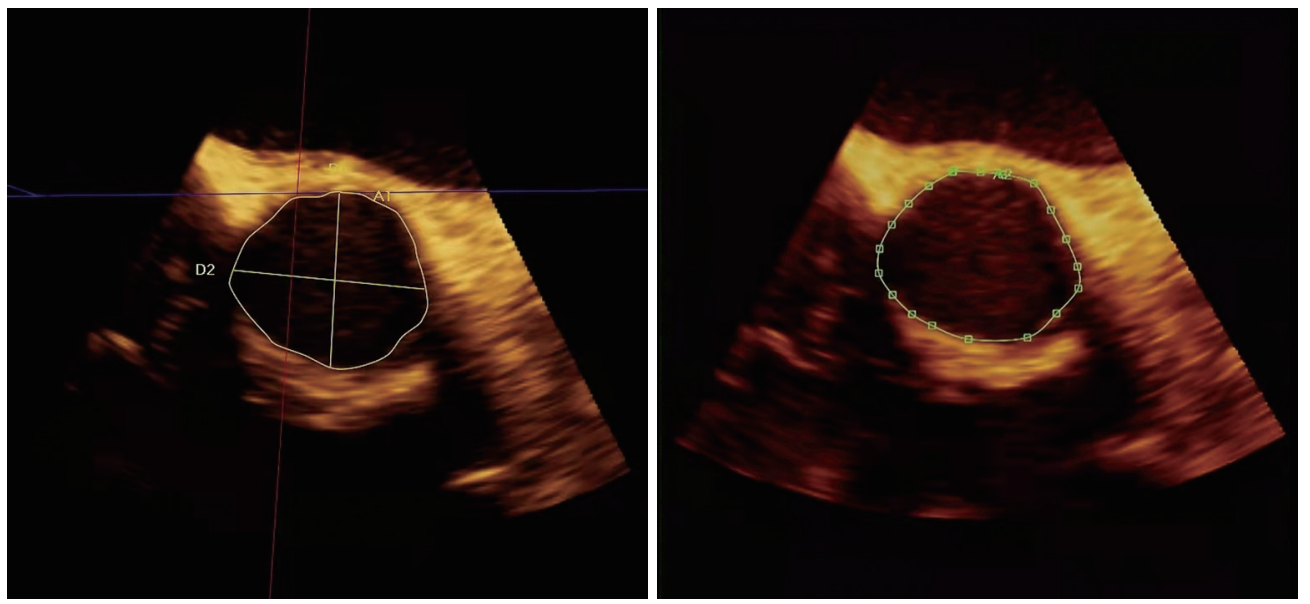

Figure 2 3D-TEE measurement of the inner diameter and area of the aortic valve annulus. 3D-TEE, three-dimensional transesophageal echocardiography.

Table 3 The general condition of the patients

\begin{tabular}{lc}
\hline Variables & Value \\
\hline Age (years) & $68.11 \pm 7.38$ \\
Male, $\mathrm{n}(\%)$ & $48(64.9)$ \\
Aortic valve stenosis, $\mathrm{n}(\%)$ & $26(35.1)$ \\
Aortic valve insufficiency, n (\%) & $28(37.8)$ \\
Aortic valve stenosis combined with aortic & $20(27.1)$ \\
insufficiency, n (\%) & $23(31.1)$ \\
Bicuspid aortic valve, n (\%) & $23(31.1)$ \\
Valve calcification, $\mathrm{n}(\%)$ & $45.05 \pm 13.54$ \\
LVEF (\%)
\end{tabular}

LVEF, left ventricular ejection fraction.

and 3D-TEE measurements. If the patient has a bicuspid aortic valve, it is necessary to select a prosthetic valve that is 1 size smaller than the predicted size (6). Postoperatively, MSCT measurements were obtained with the permission of the MSCT doctor, and a comparative study was performed (Figures 1,2).

\section{Statistical analysis}

SPSS 26.0 (SPSS Inc. Chicago, IL, USA) software was used for statistical analyses. The Kolmogorov-Smirnov test was used to test whether the quantitative data conformed to a normal distribution. The mean \pm standard deviation was used for quantitative data conforming to a normal distribution, and count data were expressed as cases or percentages. The paired-samples $t$-test was used to compare the data measurements of MSCT and 3D-TEE. The consistency of the MSCT and 3D-TEE data measurements was analyzed by the intraclass correlation coefficient (ICC). The model was chosen as a two-way random model, the method was absolute agreement, and the output results were chosen as single measurements. The accuracy of the predicted valve size between MSCT and 3D-TEE was compared by the McNemar-Bowker test, and a $\mathrm{P}$ value $<0.05$ was considered statistically significant.

\section{Results}

All patients underwent successful TAVI with the aid of 3D-TEE, including 37 with the Venus Medtech A-valve and 37 with the J-valve. All 74 patients were successfully implanted with prosthetic valves, and there were no cases of death, third-degree atrioventricular (AV) block, myocardial infarction, macrovascular events, or renal failure at the 1-year postoperative follow-up. Echocardiography at 1-year follow-up showed that EF improved in all patients compared with pre-operation $(46.81 \% \pm 15.24 \%$ vs. $54.53 \% \pm 8.69 \%$ in the $\mathrm{A}$-valve group, $\mathrm{P}<0.05$; $43.39 \% \pm 11.67 \%$ vs. $47.42 \% \pm 10.52 \%$ in the J-Valve group, $\mathrm{P}<0.05)$. The mean pressure gradient of the aortic valve decreased significantly in the A-valve group $48.47 \pm 16.10$ vs. $14.11 \pm 7.60 \mathrm{mmHg}, \mathrm{P}<0.001$, while in the J-valve group there was no significant change, all within the normal range $(12.50 \pm 15.63$ vs. $9.03 \pm 5.18 \mathrm{mmHg}, \mathrm{P}=0.14)$. A total of 19 patients had perivalvular leaks, among which 12 had trace or mild regurgitation, 7 had mild to moderate or moderate 
Table 4 3D-TEE and MSCT assessment of the aortic valve annulus

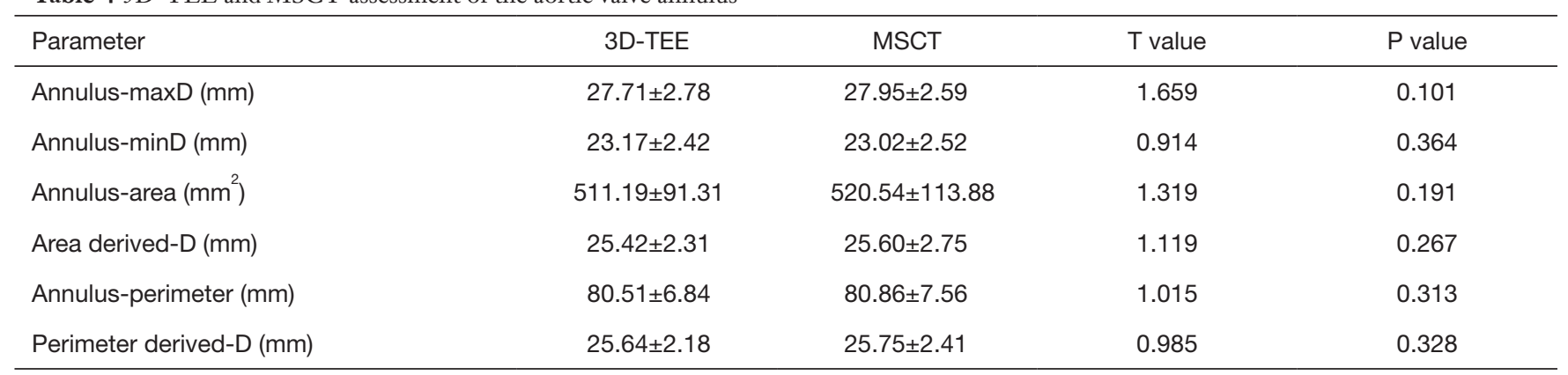

3D-TEE, three-dimensional transesophageal echocardiography; MSCT, multilayer spiral computed tomography.

Table 5 Consistency analysis of 3D-TEE and MSCT assessments of the aortic valve annulus size

\begin{tabular}{lccc}
\hline Parameter & Number of samples (case) & ICC (95\% CI) & P value \\
\hline Annulus-maxD & 74 & $0.89(0.83-0.93)$ & $<0.001$ \\
Annulus-minD & 74 & $0.83(0.75-0.89)$ & $<0.001$ \\
Area derived-D & 74 & $0.84(0.76-0.89)$ & $<0.001$ \\
Perimeter derived-D & 74 & $0.92(0.87-0.94)$ & $<0.001$ \\
\hline
\end{tabular}

3D-TEE, three-dimensional transesophageal echocardiography; MSCT, multilayer spiral computed tomography; ICC, intraclass correlation coefficient.

regurgitation, 1 had pericardial effusion immediately after surgery, and 1 had femoral artery entrapment, all of which improved after symptomatic treatment. The basic information of the patients is shown in Table 3.

There were no significant differences between the 3D-TEE and MSCT measurements in terms of the maximum diameter, minimum diameter, area, perimeter, area-derived diameter, and perimeter-derived diameter of the aortic annulus (all $\mathrm{P}>0.05$ ) (Table 4).

The consistency of the maximum diameter, minimum diameter, area-derived average diameter, and perimeterderived average diameter of the aortic valve annulus measured by $3 \mathrm{D}$-TEE and MSCT was analyzed, and the consistency correlation coefficients were $0.89,0.83,0.84$, and 0.92, respectively (Table 5, Figure 3).

3D-TEE was used to evaluate the aortic valve annulus of patients implanted with the Venus Medtech A-valve, and the recommended valve size was selected according to the CT measurement results. Two patients with an average 3D-TEE diameter of 17-19.9 mm was implanted with $23^{\#}$ valves, which was the same as the predicted result. Four patients had a mean diameter of 20-22.9 mm, 2 of whom had bicuspid aortic valves requiring 1 size down. Thus, for the implantation of 2 valves of $26^{\#}$ and 2 valves of $23^{\#}$, the actual implantation was the same as predicted. Twentyone patients had a mean diameter of $23-25.9 \mathrm{~mm}, 13$ of whom had bicuspid valves requiring 1 size down. Thus, 8 with predicted implantation of $29^{\#}$ valves and 13 with $26^{\#}$ valves. Of these patients, 4 were actually implanted with $29^{\#}$ valves, 16 with $26^{\#}$ valves, and 1 with a $23^{\#}$ valve. There were ten patients with an average diameter of $26-29 \mathrm{~mm}$, and 5 of them had bicuspid valves requiring 1 size down. It was predicted that 5 cases would be implanted with $32^{\#}$ valves and 5 cases would be implanted with $29^{\#}$ valves, and we actually implanted $29^{\#}$ valves in 3 cases and $26^{\#}$ valves in 7 cases (Table 6).

3D-TEE was used to evaluate the aortic valve annulus of patients implanted with the J-valve, and the recommended valve size was selected according to the CT measurement results. In 1 patient with an average 3D-TEE diameter of 19-20.9 $\mathrm{mm}$, the predicted and actual implanted valves were ${ }^{\# 2} 1$ valves. The average diameter was $23-24.9 \mathrm{~mm}$ in 7 patients, and these 7 were predicted to have $25^{\#}$ valves implanted. Actual implantation of the $25^{\#}$ valve was performed in 2 cases and implantation of the $27^{*}$ valve was performed in 5 cases. The mean diameter was $25-26.9 \mathrm{~mm}$ in 18 patients, including 2 with bicuspid valves. Sixteen patients were predicted to have $27^{\#}$ valves implanted, and 2 

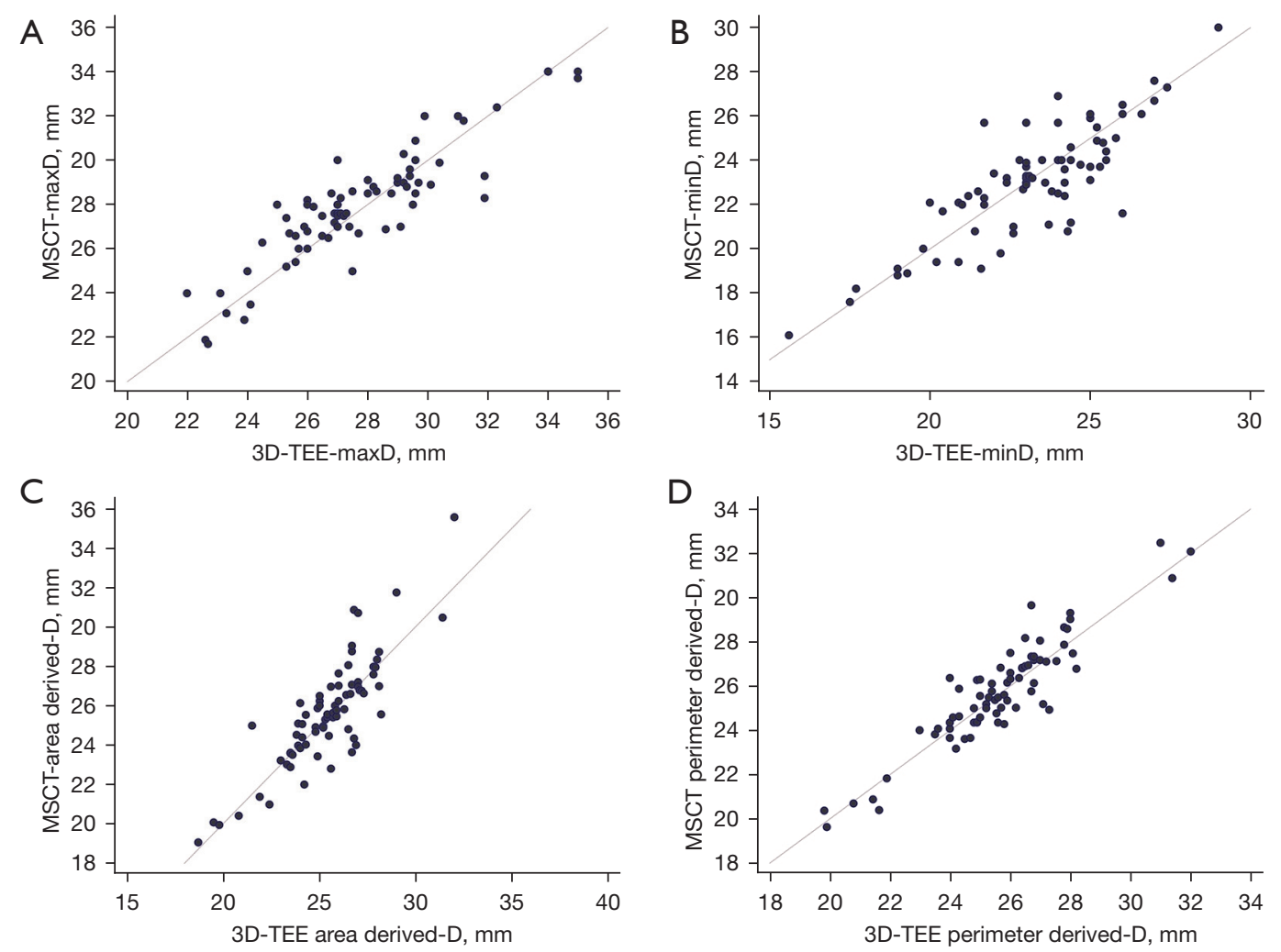

Figure 3 Scatter plots of the consistency of 3D-TEE and MSCT in assessing the aortic valve annulus size. (A-D) The 2 methods have a good correlation in measuring the maximum diameter, minimum diameter, area-derived average diameter, and perimeter-derived average diameter of the aortic annulus, respectively. 3D-TEE, three-dimensional transesophageal echocardiography; MSCT, multilayer spiral computed tomography.

Table 6 Comparison table of 3D-TEE-predicted valve size and actual implanted valve size (Venus Medtech A-valve)

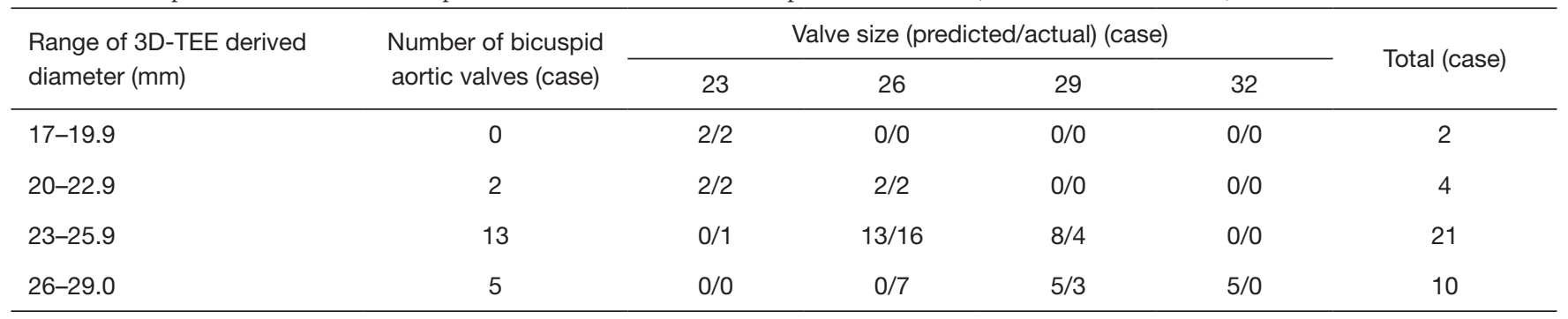

3D-TEE, three-dimensional transesophageal echocardiography.

had $25^{\#}$ valves implanted. Actual implantation of valve " 25 was performed in 2 cases, valve 27 in 10 cases, and valve ${ }^{\#} 29$ in 6 cases. There were 11 patients with an average diameter of 27-29 mm, among which 1 had a bicuspid valve. It was predicted that 1 case would be implanted with valve ${ }^{*} 27$ and 10 cases would be implanted with valve \#29, which was consistent with the actual implanted valve size (Table 7).
Statistical methods were used to comparison of the differences in the ability of MSCT and 3D-TEE to predict the prosthetic valve size. Compared with the actual implanted valve size, the matching success rate was $75.68 \%$ by MSCT and $70.27 \%$ by 3D-TEE. For the Venus Medtech A-valve, the prosthetic aortic valve size predicted by MSCT matched the actual implant in 27 cases and did 
Table 7 Comparison table of the 3D-TEE-predicted valve size and the actual implanted valve size (J-valve)

\begin{tabular}{lcccccc}
\hline \multirow{2}{*}{$\begin{array}{l}\text { Range of 3D-TEE derived } \\
\text { diameter (mm) }\end{array}$} & \multirow{2}{*}{$\begin{array}{c}\text { Number of bicuspid } \\
\text { aortic valves (case) }\end{array}$} & \multicolumn{5}{c}{ Valve size (predicted/actual) (case) } \\
\cline { 3 - 6 } Total (case)
\end{tabular}

3D-TEE, three-dimensional transesophageal echocardiography.

A

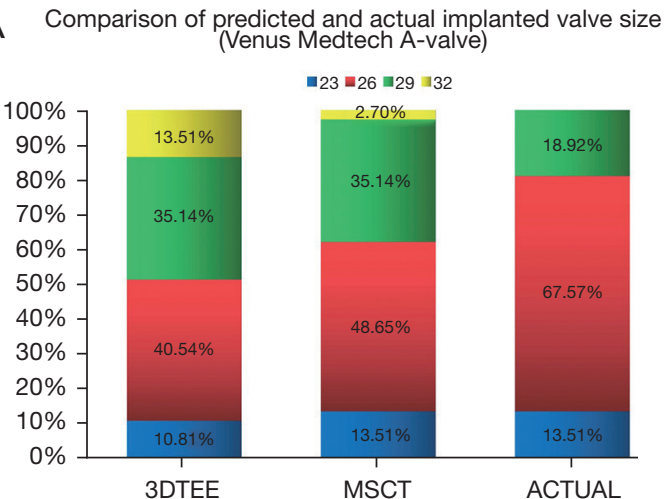

B Comparison of predicted and actual implanted valve size (J-valve)

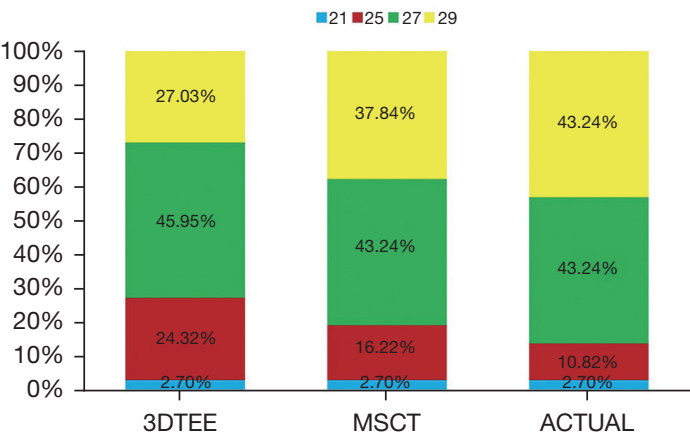

Figure 4 Comparison of 3D-TEE- and MSCT-predicted valve sizes and actual implanted valve sizes. (A) The Venus Medtech A-valve; (B) the J-valve. 3D-TEE, three-dimensional transesophageal echocardiography; MSCT, multilayer spiral computed tomography.

not match in 10 cases, and those predicted by $3 \mathrm{D}$-TEE matched in 23 cases and did not match in 14 cases $\left(\chi^{2}=7.000\right.$, $\mathrm{P}=0.136)$. For the $\mathrm{J}$-valve, the prosthetic aortic valve size predicted by MSCT matched the actual implant in 29 cases and did not match in 8 cases, and those predicted by 3D-TEE matched in 26 cases and did not match in 11 cases $\left(\chi^{2}=4.467, \mathrm{P}=0.107\right)$. There was no significant difference in the prediction accuracy of the 2 methods for different prosthetic valve sizes in TAVI surgery (Figure 4).

\section{Discussion}

With the development of TAVI technology, an increasing number of patients with aortic valve disease listed as having contraindications or being high-risk for surgical operations tend to choose TAVI surgery. Accurate assessment of the aortic annulus is an important guarantee of successful surgery and reduced postoperative complications (7). In this study, MSCT measurements were applied intraoperatively to guide the selection of prosthetic valves. All patients underwent TAVI successfully, and no serious complications, such as death, third-degree AV block, myocardial infarction, or macrovascular events, were found after surgery. After the 1-year follow-up, cardiac function improved significantly in all patients. The pressure gradient of aortic valves had a significant reduction in patients with aortic stenosis, but no increased in patients with aortic valve insufficiency due to the implantation of a new valve. A total of 19 patients had perivalvular leaks after surgery, among which 7 were mild to moderate, and no severe perivalvular leaks occurred. It has been shown that the size of the calcification volume plays a key role in the occurrence of perivalvular leakage after TAVI (8). In the present study, 1 case of moderate perivalvular leakage occurred with a mildly tilted prosthetic valve, while the rest had AS, and more severe calcification was seen. The source, number, and severity of perivalvular leaks can be observed with TEE immediately after surgery to analyze whether poor valve shaping due to calcification is causing the perivalvular leaks, which can be addressed with balloon dilation, implantation of a "valve in valve", or 
modification of the valve type.

At present, imaging modalities that can be used for preoperative evaluation of TAVI include CT, 3D-TEE and $3 \mathrm{D}$ rotational angiography (9). Considering the ease of operation and image clarity, the most commonly used clinical technique is CT examination, although prospective randomized controlled trials for MSCT have never been perform (10). While 3D-TEE, due to its clear images, easy to operated and can be used real-time for continuous intraoperative monitoring, therefore, it not only plays an important role in the evaluation of valve function after TAVI but also allows for real-time guidance of prosthetic valve release during TAVI, preoperative assessment of aortic leaflet morphology and function, and the measurement of aortic root structures. Among them, the research of $3 \mathrm{D}$-TEE in the accuracy of aortic root measurement before TAVI has become a hot topic of research in recent years. Many previous studies have shown that 3D-TEE is correlated and consistent with CT in the measurement of the aortic valve annulus, even calcification of the aortic annulus diameter was not found to exert a noteworthy negative influence on measurements for standardized TEE at good image quality. However, it is believed that the diameter of the aortic annulus measured by TEE are usually smaller than CT, with exception of sagittal diameters. However, the feasibility and accuracy of applying 3D-TEE to select prosthetic valve size is unclear, and there have been no reports of successful TAVI with 3D-TEE measurements applied alone (10-14). This study also found a strong agreement between the 2 methods. In addition, we also investigated the ability of 3D-TEE to predict the size of different types of self-expanding prosthetic valves in aortic stenosis and aortic insufficiency, when using different surgical approaches (transfemoral and transapical approaches). The valve size predicted by both methods was similar to the actual implanted valve size, suggesting that 3D-TEE may have similar results to MSCT for the selection of TAVI valve size.

The cases implanted with the Venus Medtech A-valve were all patients with AS, 11 of whom had combined aortic regurgitation, 21 of whom had calcification, and $20(54.05 \%)$ of whom had bicuspid aortic valves. It has been demonstrated that patients with a bicuspid valve undergoing TAVI have similar benefits to those with a trilobular aortic valve but they are less successful in terms of procedural success and residual leakage $(15,16)$. Currently, echocardiography is the preferred method for diagnosing aortic valves, with a sensitivity of $87 \%$ and specificity of $95-$
$96 \%$ for a bicuspid valve $(17,18)$. 3D-TEE can display the aortic valve morphology in $3 \mathrm{D}$, clarify the type of bicuspid aortic valve, and achieve a detection rate of $100 \%$ for bicuspid valves $(19,20)$. In this study, TEE detected type 0 bicuspid aortic valves in 3 cases, type 1 in 20 cases, and type 2 in 0 cases. Among the type 1 cases, the number of cases with left and right coronary valve fusion was 15, 4 cases had non-coronary valve and right coronary valve fusion, and 1 case had non-coronary valve and left coronary valve fusion. TEE also clearly shows the distribution of the calcification, the location of the valve orifice, and the presence of eccentric opening. Real-time images can help to predict the location of the prosthetic valve after implantation and the possible sites of perivalvular leakage intraoperatively, which helps in the selection of the prosthetic valve. Because the bicuspid valve often has an elliptical annulus, severe and asymmetric valve calcification, unequal leaflets, a widened ascending aortic internal diameter, and a leaflet opening smaller than the annular internal diameter, these pathological changes are likely to result in a poor TAVI valve implantation position, poor valve stent expansion, and perivalvular residual leakage. To address these issues, some experts have proposed "supra-annular structure assessment", "supra-annular sizing", and "reshaping TAVR" $(6,21,22)$, whose main idea is to select the valve size based on the leaflet structure and recommended valve size in terms of the annular size to prevent poor valve expansion and rupture of the aortic root structure due to oversized prosthetic valves. Based on the aforementioned suggestion, the present study reduced the intraoperative selection of the prosthetic valve size for the bicuspid aortic valve by 1 valve size compared with the measured annular diameter, and the actual intraoperative implantation of the valve will take into account additional factors in selecting the valve size. However, its long-term efficacy remains to be studied.

Among the patients implanted with the J-valve, 11 patients were implanted with a valve 1 size larger than predicted. In this group of cases, severe aortic regurgitation was the main problem, usually without valve calcification, and in some patients marked dilatation of the aortic root was observed. After prosthetic valve implantation, the prosthetic valve mainly relies on the positioning members of the prosthetic valve itself and the radial support of the annulus to exert a fixation effect. When the aortic root is dilated, this can lead to a reduction in the prosthetic valve anchorage zone, increasing the risk of valve implantation failure. It has been shown that in patients with TAVI who have a significantly dilated aortic root, a slightly 
larger prosthetic valve is recommended to ensure that adequate anchorage can be achieved to minimize the risk of perivalvular leakage $(23,24)$. However, there is no uniform standard for the degree of enlargement of the prosthetic valve size selected for patients with dilated aortic roots. Therefore, in this study, the predicted valve size was strictly based on the MSCT and 3D-TEE measurements, with correction for downsizing among patients with bicuspid aortic valves, without including other influencing factors such as significant dilatation of the aortic root and calcification. This resulted in the implantation of a larger prosthetic valve than the predicted type in 11 patients. This subset of patients was also the main reason for the low compliance between the predicted and actual implant size by MSCT and 3D-TEE. This also suggests that in addition to measuring the aortic annular internal diameter, other influencing factors should be taken into account when selecting valve size. But this did not affect the consistency of 3D-TEE with MSCT for predicting the appropriate prosthetic valve size.

\section{Limitations}

The sample size of this study was limited, and the factors affecting the valve size were not comprehensively analyzed, and the analysis was retrospective, which may cause deviations in the results. In the future, a multicenter format with an expanded sample size and longer-term clinical observation of patients would help the accuracy of the results. In addition, $3 \mathrm{D}$-TEE is more dependent on the operator level and experience than CT, and it is still not a substitute for CT for measuring the distance between the annulus and coronary opening, leaflet length, ascending aortic diameter, and assessing the severity of calcification, as well as the quality of the access vessels. Severe calcification of the aortic annulus and surrounding tissue can affect the image quality of $3 \mathrm{D}$-TEE, blurring the boundaries of the annulus and leading to inaccurate measurements. In addition, TEE may induce arrhythmias, especially in extremely weak patients, and is also contraindicated in patients with esophageal varices. Therefore, although we believe that 3D-TEE can be used in place of CT in the selection of TAVI valves in special cases, its selection criteria still need further investigation. At present, the simultaneous use of both methods and their complementary use are recommended for patients who can undergo MSCT, which helps to select the valve size more precisely to reduce the incidence of postoperative complications (25).

\section{Conclusions}

In summary, there is strong agreement between 3D-TEE and MSCT for measuring the annular dimensions before TAVI in patients with severe AS and AI. The predictive power of the prosthetic valve size based solely on the mean diameter measured by both methods was equal. Obtaining an accurate mean annular diameter is the most important factor in applying 3D-TEE to guide prosthetic valve selection for TAVI surgery, but an overall assessment of the type of disease, morphology, and function of the valve leaflets, the degree of calcification, the supravalvular and subvalvular structures of the aortic valve, and the patient's cardiac function can affect the valve size selection and require over- or downsizing depending on the situation. In addition, because of its real-time nature, 3D-TEE plays an important role both intraoperatively and postoperatively. Therefore, 3D-TEE can be not only a viable alternative to CT but also a complementary technique to CT in TAVI surgery.

\section{Acknowledgments}

Funding: The study was supported by National Natural Science Foundation of China (grant number 82071932), Innovation Capability Support Program of Shaanxi Province (grant number 2020TD-034), and National Key Research and Development Program of China for Proactive Health and Technology Response to Aging in 2021Research on Standard Assessment System and Optimized Treatment Path for Geriatric Valve Disease (grant number 2020YFC2008100).

\section{Footnote}

Reporting Checklist: The authors have completed the STROBE reporting checklist. Available at https://atm. amegroups.com/article/view/10.21037/atm-21-6577/rc

Data Sharing Statement: Available at https://atm.amegroups. com/article/view/10.21037/atm-21-6577/dss

Conflicts of Interest: All authors have completed the ICMJE uniform disclosure form (available at https://atm. amegroups.com/article/view/10.21037/atm-21-6577/coif). The authors have no conflicts of interest to declare.

Ethical Statement: The authors are accountable for all 
aspects of the work in ensuring that questions related to the accuracy or integrity of any part of the work are appropriately investigated and resolved. All procedures in this study involving human participants were performed in accordance with the Declaration of Helsinki (as revised in 2013). This study was approved by the ethics board of Xijing Hospital (approval No. QX20191043-1). All patients provided written informed consent to participate in the study.

Open Access Statement: This is an Open Access article distributed in accordance with the Creative Commons Attribution-NonCommercial-NoDerivs 4.0 International License (CC BY-NC-ND 4.0), which permits the noncommercial replication and distribution of the article with the strict proviso that no changes or edits are made and the original work is properly cited (including links to both the formal publication through the relevant DOI and the license). See: https://creativecommons.org/licenses/by-nc-nd/4.0/.

\section{References}

1. Kheiri B, Osman M, Bakhit A, et al. Meta-Analysis of Transcatheter Aortic Valve Replacement in Low-Risk Patients. Am J Med 2020;133:e38-41.

2. Barbanti M, Petronio AS, Ettori F, et al. 5-Year Outcomes After Transcatheter Aortic Valve Implantation With CoreValve Prosthesis. JACC Cardiovasc Interv 2015;8:1084-91.

3. Rong LQ, Hameed I, Salemi A, et al. Three-Dimensional Echocardiography for Transcatheter Aortic Valve Replacement Sizing: A Systematic Review and MetaAnalysis. J Am Heart Assoc 2019;8:e013463.

4. Vaquerizo B, Spaziano M, Alali J, et al. Three-dimensional echocardiography vs computed tomography for transcatheter aortic valve replacement sizing. Eur Heart J Cardiovasc Imaging 2016;17:15-23.

5. Zhang M, Wan L, Liu K, et al. Aortic roots assessment by an automated three-dimensional transesophageal echocardiography: an intra-individual comparison. Int J Cardiovasc Imaging 2019;35:2029-36.

6. Liu X, He Y, Zhu Q, et al. Supra-annular structure assessment for self-expanding transcatheter heart valve size selection in patients with bicuspid aortic valve. Catheter Cardiovasc Interv 2018;91:986-94.

7. Blanke P, Willson AB, Webb JG, et al. Oversizing in transcatheter aortic valve replacement, a commonly used term but a poorly understood one: dependency on definition and geometrical measurements. J Cardiovasc Comput Tomogr 2014;8:67-76.

8. Guez D, Boroumand G, Ruggiero NJ, et al. Automated and Manual Measurements of the Aortic Annulus with ECG-Gated Cardiac CT Angiography Prior to Transcatheter Aortic Valve Replacement: Comparison with 3D-Transesophageal Echocardiography. Acad Radiol 2017;24:587-93.

9. Meyhōer J, Ahrens J, Neuss M, et al. Rotational angiography for preinterventional imaging in transcatheter aortic valve implantation. Catheter Cardiovasc Interv 2012;79:756-65.

10. Fox H, Hemmann K, Lehmann R. Comparison of transthoracic and transesophageal echocardiography for transcatheter aortic valve replacement sizing in high-risk patients. J Echocardiogr 2020;18:47-56.

11. Hammerstingl C, Schueler R, Weber M, et al. Threedimensional imaging of the aortic valve geometry for prosthesis sizing prior to transcatheter aortic valve replacement. Int J Cardiol 2014;174:844-9.

12. Kato N, Shibayama K, Noguchi M, et al. Superiority of novel automated assessment of aortic annulus by intraoperative three-dimensional transesophageal echocardiography in patients with severe aortic stenosis: Comparison with conventional cross-sectional assessment. J Cardiol 2018;72:321-7.

13. Kochman J, Rymuza B, Huczek Z. Transcatheter aortic valve replacement in bicuspid aortic valve disease. Curr Opin Cardiol 2015;30:594-602.

14. Husser O, Holzamer A, Resch M, et al. Prosthesis sizing for transcatheter aortic valve implantation--comparison of three dimensional transesophageal echocardiography with multislice computed tomography. Int J Cardiol 2013;168:3431-8.

15. Bauer T, Linke A, Sievert H, et al. Comparison of the effectiveness of transcatheter aortic valve implantation in patients with stenotic bicuspid versus tricuspid aortic valves (from the German TAVI Registry). Am J Cardiol 2014;113:518-21.

16. Mylotte D, Lefevre T, Søndergaard L, et al. Transcatheter aortic valve replacement in bicuspid aortic valve disease. J Am Coll Cardiol 2014;64:2330-9.

17. Zhou Q, Bi BJ, Xia ZM, et al. Application value of GE Vivid E9 Doppler echocardiography in the diagnosis of two-lobar aortic valve malformation. J Integr Tradit Chin Western Med Cardio-Cerebrovasc Dis 2019;17:3187-9.

18. Kari FA, Beyersdorf F, Siepe M. Pathophysiological implications of different bicuspid aortic valve 
configurations. Cardiol Res Pract 2012;2012:735829.

19. Espinola-Zavaleta N, Muñoz-Castellanos L, Attié F, et al. Anatomic three-dimensional echocardiographic correlation of bicuspid aortic valve. J Am Soc Echocardiogr 2003;16:46-53.

20. Citro R, Cecconi M, La Carrubba S, et al. Bicuspid Aortic Valve Registry of the Italian Society of Echocardiography and Cardiovascular Imaging (REgistro della valvola aortica bicuspide della società italiana di ECocardiografia e CArdiovascular imaging): Rationale and Study Design. J Cardiovasc Echogr 2018;28:78-89.

21. Xiong TY, Feng Y, Li YJ, et al. Supra-Annular Sizing for Transcatheter Aortic Valve Replacement Candidates With Bicuspid Aortic Valve. JACC Cardiovasc Interv 2018;11:1789-90.

22. Zhao ZG, Feng Y, Liao YB, et al. Reshaping bicuspid aortic valve stenosis with an hourglass-shaped balloon for transcatheter aortic valve replacement: A pilot study.

Cite this article as: Meng X, Sun Y, Bai W, Li Y, Tuo S, Cao L, Du M, Liu Y, Jin P, Yang J, Liu L. Application research of three-dimensional transesophageal echocardiography in predicting prosthetic valve size for transcatheter aortic valve implantation. Ann Transl Med 2022;10(2):84. doi: 10.21037/ atm-21-6577
Catheter Cardiovasc Interv 2020;95 Suppl 1:616-23.

23. Alkhouli M, Sengupta P, Badhwar V. Toward Precision in Balloon-Expandable TAVR: Oversizing Tight Versus Just Right. JACC Cardiovasc Interv 2017;10:821-3.

24. Maeno Y, Yoon SH, Abramowitz Y, et al. Effect of ascending aortic dimension on acute procedural success following self-expanding transcatheter aortic valve replacement: A multicenter retrospective analysis. Int J Cardiol 2017;244:100-5.

25. Willson AB, Webb JG, Freeman M, et al. Computed tomography-based sizing recommendations for transcatheter aortic valve replacement with balloonexpandable valves: Comparison with transesophageal echocardiography and rationale for implementation in a prospective trial. J Cardiovasc Comput Tomogr 2012;6:406-14.

(English Language Editor: C. Betlazar-Maseh) 